\title{
A guide to qualitative haze measurements demonstrated on inkjet-printed silver electrodes for flexible OLEDs
}

Felix Hermerschmidt, Michael Hengge, Vincent R. F. Schröder, Paul Hänsch, Konstantin Livanov, et al.

Felix Hermerschmidt, Michael Hengge, Vincent R. F. Schröder, Paul Hänsch, Konstantin Livanov, Natalia Zamoshchik, Emil J. W. List-Kratochvil, "A guide to qualitative haze measurements demonstrated on inkjet-printed silver electrodes for flexible OLEDs," Proc. SPIE 11808, Organic and Hybrid Light Emitting Materials and Devices XXV, 118080B (9 August 2021); doi: $10.1117 / 12.2594486$

Event: SPIE Organic Photonics + Electronics, 2021, San Diego, California, United States 


\title{
A guide to qualitative haze measurements demonstrated on inkjet-printed silver electrodes for flexible OLEDs
}

\author{
Felix Hermerschmidt*a, Michael Hengge ${ }^{\mathrm{b}}$, Vincent R. F. Schröder ${ }^{\mathrm{b}}$, Paul Hänsch ${ }^{\mathrm{a}}$, \\ Konstantin Livanov ${ }^{\mathrm{c}}$, Natalia Zamoshchik ${ }^{\mathrm{c}}$, Emil J. W. List-Kratochvil ${ }^{\mathrm{a}, \mathrm{b}}$ \\ ${ }^{a}$ Humboldt-Universität zu Berlin, Institut für Physik, Institut für Chemie, IRIS Adlershof, Zum

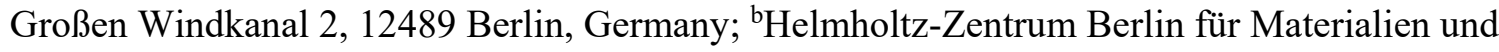 \\ Energie GmbH, Hahn-Meitner-Platz 1, 14109 Berlin, Germany; 'Oreltech GmbH, Rudower \\ Chaussee 29, 12489 Berlin, Germany
}

\begin{abstract}
The search for alternative transparent electrodes to the commonly used indium tin oxide (ITO) in optoelectronic devices has led to solution-based approaches based on inkjet printing. As an additive manufacturing technique that allows drops to be positioned only where necessary, inkjet printing shows reduced waste of starting material compared to other methods such as spin coating. As a result, functional materials can be both coated and structured without the need for masks or lithographic pre-patterning of the substrate.

For this contribution, we utilized a particle-free silver ink to produce a transparent electrode by inkjet printing. After printing, the silver ions were reduced to metallic silver by an argon plasma. The process takes place at low temperatures (ca. $40-50{ }^{\circ} \mathrm{C}$ ), making it suitable for use with flexible substrates, which are often temperature-sensitive. The printed silver layers show good electrical conductivity and optical transmittance, with a crystalline grain structure being formed and maintained during the metallization process. This structure forms a self-organized nanometer-size grid, whose structure allows light to pass through.

Due to its nano-structured property, the haze of the electrode was investigated using a simple experimental setup based on a light source shining through the electrode and analyzing the size of the projected pattern. Such qualitative assessment can be a useful indication of the quality of the electrode and we provide details on how to replicate this setup. The final electrodes were implemented in solution-processed OLEDs, which showed bright luminance and overall low haze compared to ITO-based reference devices.
\end{abstract}

Keywords: organic light-emitting devices, flexible transparent electrodes, inkjet printing, solution processing, low haze

\section{INTRODUCTION}

Many optoelectronic devices contain a transparent conducting electrode, in order to allow light to either enter or leave the device through that electrode. Indium tin oxide (ITO) is the most commonly used material for this purpose, due to its high optical transmission in the visible spectral range and its low sheet resistance. ${ }^{2}$ However, ITO is a brittle material, which can limit its use when targeting bendable applications on flexible substrates. ${ }^{34}$ As a result, alternative materials have been investigated, which predominantly rely on one of two deposition methods - vacuum-based from the solid state or solutionbased methods. ${ }^{5}$

Vacuum-based alternatives include dielectric/metal/dielectric layers and thin metal films, ${ }^{678}$ while solution-based alternatives include using conducting polymers, ${ }^{9}$ nanowires, ${ }^{10}$ nanoparticle inks, ${ }^{11}$ and more recently, particle-free inks. ${ }^{12} 131415$ The latter type of inks utilize mostly oxidized metal precursors dissolved in a suitable solvent together with additives and stabilizing agents. Therefore, shelf-life is improved, due to a reduced chance of particle agglomeration. These type of inks are particularly interesting, because they can be deposited wholly over the electrode surface with a transmission of $>70 \%,{ }^{16}$ as opposed to nanoparticle inks, which are typically deposited as lines or grids due to their opaque nature. ${ }^{17} 1819$

In order to access such structures, inkjet printing is an advantageous technique due to its drop-on-demand capability, which requires no masks or lithographic patterning of the substrate and results in great freedom of design to print arbitrary

*felix.hermerschmidt@hu-berlin.de; phone+49 302093 82524; hyd.iris-adlershof.de

Organic and Hybrid Light Emitting Materials and Devices XXV, edited by Chihaya Adachi, Tae-Woo Lee,

Franky So, Proc. of SPIE Vol. 11808, 118080B - (c) The Authors. Published under a Creative Commons Attribution CC-BY 3.0 License · doi: 10.1117/12.2594486 
patterns. ${ }^{20}{ }^{21}$ Material waste is also reduced due to its additive nature, meaning inkjet printing can be used to coat defined areas, ${ }^{22}{ }^{23}$ while being scalable and compatible with industrial printheads and processes. ${ }^{24}$

Both types of inks - nanoparticle-based or particle-free - also show a high resilience to bending once deposited. This is a parameter which is becoming increasingly important as industrial processes move towards flexible devices and large-area production. ${ }^{25}$ Bending tests can be performed in an automated fashion to repeatedly strain the sample thousands of times, ${ }^{26} 2728$ but often it is already enough to perform a few tens or hundreds of bending tests to gain information about the resilience of the sample. The resilience is indicated by the investigation of the relative resistance as a function of bending cycles. As opposed to ITO, samples containing the abovementioned alternative electrode types show only a low relative resistance increase, and are therefore well suited to flexible device implementation. ${ }^{29} 30$

In this work, a silver particle-free ink is inkjet-printed and implemented as a transparent conductive electrode on rigid glass and flexible polyethylene terephthalate (PET) substrates. Due to the nature of the morphological structure formed during sintering of the electrode, the haze of the layer was investigated. When implemented in organic light-emitting devices (OLEDs), the electrodes provide the necessary electrical and optical properties to yield devices with high brightness and low haze.

\section{INKJET-PRINTED ELECTRODES IN FLEXIBLE OLEDS}

The silver ink OTech T 1053 was used to fabricate the electrodes investigated in this study. The ink is suitable for inkjet printing since its viscosity and surface tension are optimized for this application (ca. $10 \mathrm{cP}$ and ca. $25 \mathrm{mN} / \mathrm{m}$, respectively). After inkjet printing the electrode layer, the ink is subjected to mild argon plasma irradiation, which leads to the reduction of $\mathrm{Ag}^{+}$to $\mathrm{Ag}^{0}$ and the agglomeration to particles, which in turn form a metallic silver layer ${ }^{31}$ The process takes place at low temperatures (ca. $40-50^{\circ} \mathrm{C}$ ), making it suitable for use with flexible substrates, which are often temperature-sensitive. the exact process is described in our previous work. ${ }^{32}$

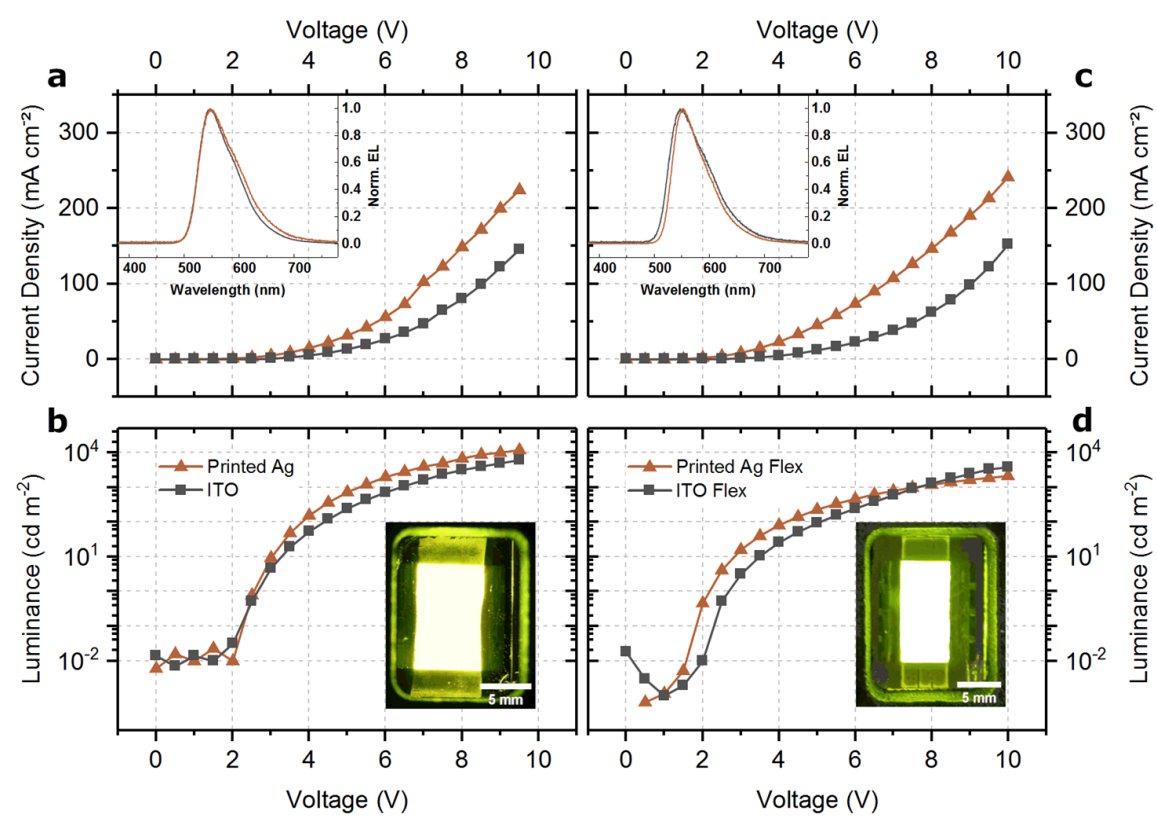

Figure 1. The device performance of the OLEDs fabricated on the inkjet-printed silver electrode is superior to those based on ITO, both on glass (a, b) and on flexible PET (c, d). The luminance is superior in the case of glass (b), while on flexible devices, higher brightness is seen in the low voltage range up to approximately $7 \mathrm{~V}$. The insets of the electroluminescence spectra and photographs of the devices in operando confirm the yellow light emitted at $1000 \mathrm{~cd} \mathrm{~m}^{-2}$. Modified from Hengge et al. ${ }^{32}$ 
When now implementing these electrodes in solution-processed OLEDs on both rigid glass and flexible PET substrates using a commercially available polymer (Super Yellow) as the emissive layer, the devices show excellent performance (see Figure 1). Firstly, the current density / voltage characteristics show similar current densities at the start of the voltage sweep. However, upon increasing voltage, the current density of the OLEDs incorporating the inkjet-printed Ag layer increase more rapidly than the ITO-based reference devices (Figure 1a, c). This is most likely due to the lower sheet resistance of the Ag layer. Secondly, the luminance characteristics of the OLEDs incorporating printed Ag show brighter devices than those incorporating ITO. This is the case from turn-on (voltage at which luminance $\geq 1 \mathrm{~cd} \mathrm{~m}^{-2}$ ) for both rigid glass and flexible PET substrates. At high voltages the flexible devices incorporating ITO reach higher absolute values.

One of the reasons for the high performance of the transparent electrode based on the inkjet-printed ink is its good interplay between electrical conductivity and optical transmission. Particularly interesting in this regard is the distinct crystalline structure that is formed upon drying and throughout the metallization process of the electrode. The structure influences the electrical properties, since more closed and connected features enhance conductivity, while also influencing the light transmission, since more open features allow light to pass through. Figure 2 shows a surface force and optical microscopy image of the structure in a fully dried and metallized film, whose needle-like features are visible down to a small scale.

Since such crystalline structures can have an impact on the scattering effect of light passing through it, it was imperative to investigate this structure further. In particular the haze of the layer in combination with the substrate provides further insight into the optical behavior of the transparent electrodes.
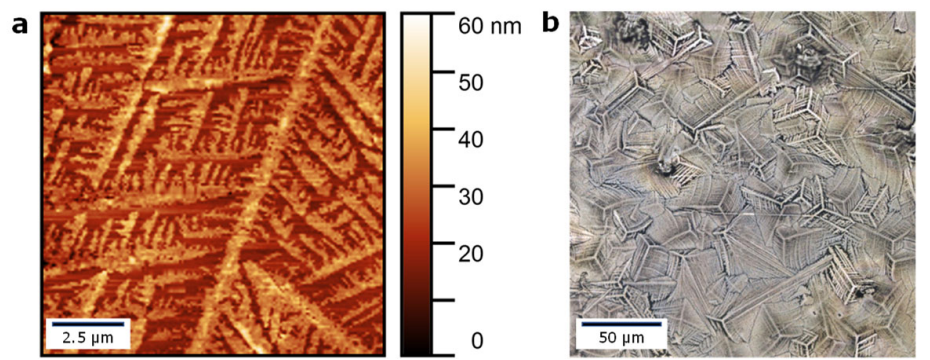

Figure 2. The inkjet-printed layer of Ag ink shows a distinct, not fully closed, crystalline structure, visible with both surface force microscopy (a) and optical microscopy (b). Investigating the effect of this structure on the haze of the sample led us to develop the setup described in this paper. Modified from Hengge et al. ${ }^{32}$

\section{HAZE}

As a beam of light is incident on a (transparent) film, the light can be transmitted through the layer, reflected at the front surface, reflected at the back interface or scattered by the film. The amount of scattering an incident beam experiences is called haze in case it is scattered by more than $2.5^{\circ}$, and it is called clarity in case it is scattered less than $2.5^{\circ}$ from the normal of the initial point source of the light. Quantifying haze is important for any applications on transparent films which require a viewer to observe a clear image.

The measurement of haze is governed by two test standards - ASTM D1003 and BS EN ISO $12468 .{ }^{33} 34$ These standards describe the measurement of haze either with a spectrophotometer or a dedicated hazemeter. In both cases the total haze is calculated from the ratio of the diffuse transmittance to the total transmittance. While the result of this type of haze measurement yields quantitative results, it requires specialized pieces of equipment and particular setups.

In the case of a spectrophotometer, a specific set of measurements need to be carried out in order to fulfil the standard. Four separate scans are required through an integrating sphere. Firstly, a white standard is placed at the exit of the integrating sphere, secondly a sample is placed at the entrance of the sphere, keeping the white standard in place. Thirdly, both sample and white standard are removed, and lastly only the sample is left and the light is left to be scattered around the integrating sphere. The haze value can then be calculated according to a formula that combines the integrals of each of the four measurements. The full procedure is described in detail elsewhere. ${ }^{35} 36$

In the case of a hazemeter, the procedure is simpler, since the piece of equipment and the corresponding ports are designed to measure haze specifically. The integrating sphere contains a ring detector which can immediately detect the light deviating from the normal incidence path. ${ }^{37}$ Huang et al. use such a hazemeter to determine the scattering properties of a 
poly methyl methacrylate (PMMA) sample. ${ }^{38}$ By shining green light through the sample, the authors observed an increase in spot size of the light beam compared to without the sample, indicative of a diffusion of the beam. Inspired by this work, we provide here a simple qualitative experimental approach to systematically determine the haze of a range of transparent films without the need for a dedicated piece of equipment. The setup is designed to be built from readily accessible parts by shining a laser pointer through a sample and observing the pattern formed on a target. Depending on the size of the spot that is created on the target pattern, it is possible to obtain a qualitative haze measurement, i.e. whether a sample is more or less hazy than a reference.

\section{EXPERIMENTAL SETUP}

The haze experiments we are describing in this paper are based on a very simple setup, consisting of components readily available in the laboratories of the majority of chemistry and physics research groups. A printout of a target pattern is fixed onto a free wall. At a distance of $1 \mathrm{~m}$ from the target, the sample to be investigated is placed in a holder. The holder should be affixed, so that the sample is in line with the center of the target. A laser pointer is then held in place by a screw clamp, with the end of the laser pointer $10 \mathrm{~mm}$ away from the sample, also in line with the target. The screw clamp can be used to directly press the operating button of the laser pointer.

a

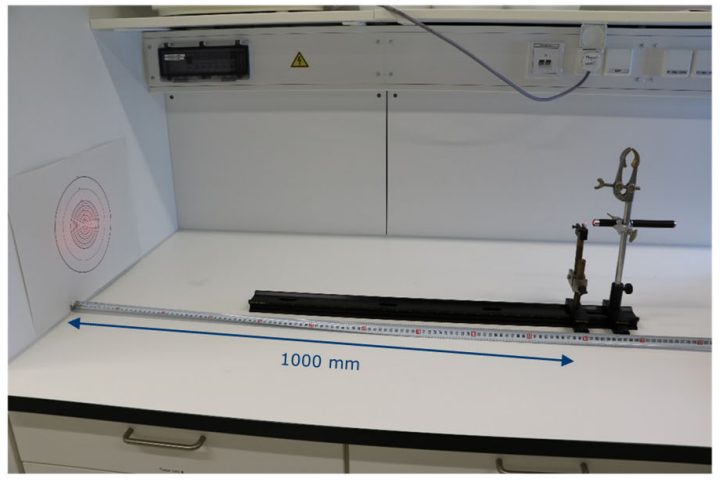

C

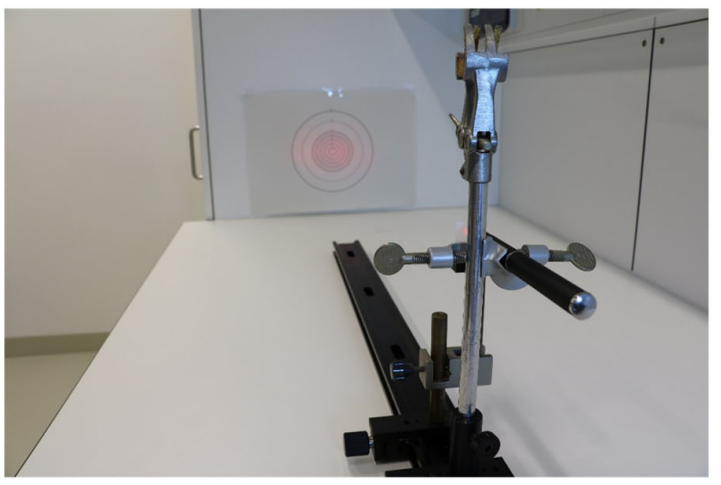

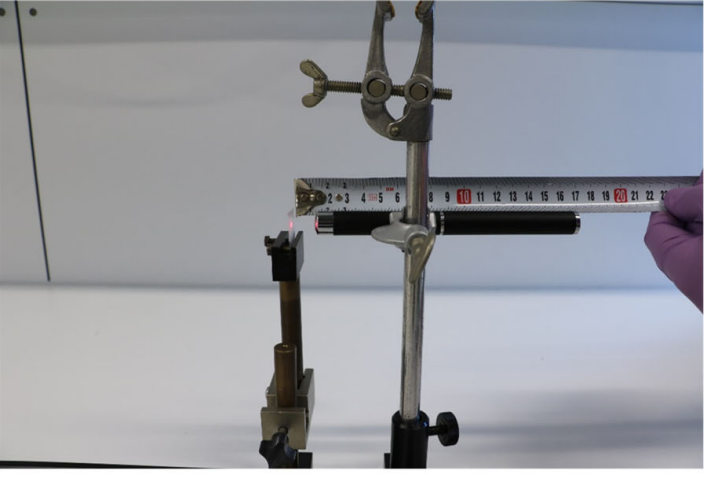

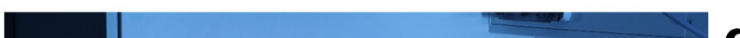

Figure 3. The sample is positioned $100 \mathrm{~cm}$ from the target (a), while the laser pointer is held in place at a distance of $1 \mathrm{~cm}$ from the sample (b). The setup consists of components available in most experimental research groups and can easily be substituted by other parts that fulfil the same purpose (c). When the laser light shines through the sample, a pattern is formed on the target, whose size depends on the haze of the sample (d).

Figure 3 shows photographs of our setup. It consists of individual components we had access to from an optics laboratory. The most important component is the laser pointer, though all measurements should be done using the same color pointer for comparison reasons. Any type of laser pointer will do as long as a system is found to depress the button, even if it is manually. In order to carry out your own experiments, it is possible to use alternative pieces of equipment that fulfil the same purpose. Even the distance values can be altered, however the resulting angles would then need to be recalculated.

The precise list of items used for our setup consists of the following: 
- Laser pointer of any color. Alternatively, a laser diode can be used, but most research groups have a laser pointer readily available. Preferably the laser pointer has an easily depressable button, but any other is also ok.

- Screw clamp to hold laser pointer in place and to operate it.

- Clamp on which the laser pointer clamp is held.

- Adjustable foot, which holds the clamp and sits on the guiding rail.

- Guiding rail in order to adjust the distances of sample and laser pointer from each other and from the target. Alternatively, these distances can be set up directly at the specific distance points.

- Sample holder. In our case this was a clamp which held the sample in place with a screw. Any alternative holder will do, as long as it can hold the sample in place and upright in front of the laser pointer opening.

- Adjustable foot, which holds the sample holder on the guiding rail.

- A3 printout of target circles. We have included a design within this paper to be printed out 1:1. It is recommended to print on A3 in order to fit slightly more circles on the design, but an A4 printout is fine also.

- $\quad$ Adhesive tape to attach the paper to a wall.

- Camera and (optional) tripod.

Start by sticking the paper printout of the target circles onto a wall. Align the laser pointer with the center point of the target by using the clamps. The sample should now be mounted on the sample holder in between laser pointer and target, at a distance of $1000 \mathrm{~mm}$ from the target and $10 \mathrm{~mm}$ from the opening of the laser pointer. Use a screw clamp in such a way as to depress the button to operate the laser pointer, so that it does not need to be pressed manually. Set up the camera on a tripod to be able to take sharp images of the pattern without any blur and shadow of the camera. Fix the exposure time, lens speed and ISO value to obtain comparable pictures, especially since the images will be taken in low light conditions. An angle can then be calculated from the radius of the image pattern at the given distance away from the sample surface (see Figure 4), in our case $1000 \mathrm{~mm}$.

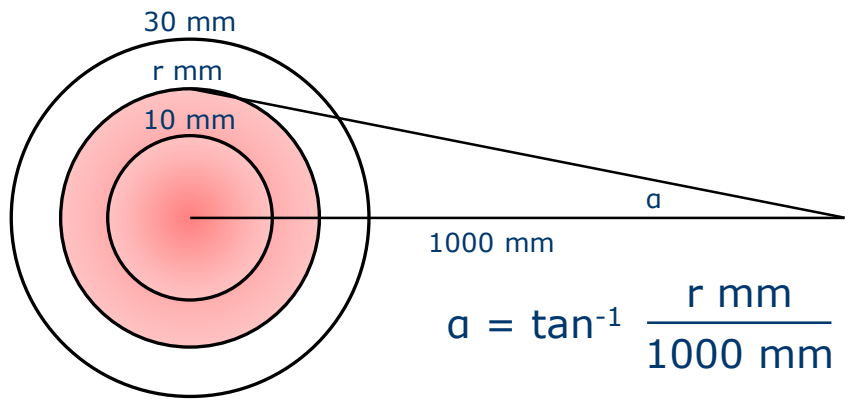

Figure 4. The angle of the pattern can be calculated trigonometrically from the given distances and thereby provide information on the haze.

A final note on this setup. At its most basic, the setup simply requires a laser pointer, the sample and a target, which can each be held manually. In order to make the measurements more accurate, we would recommend clamping/holding each of these parts in place, which is how we developed our setup. Nevertheless, the setup can be changed according to the available components in your lab.

The samples measured in this setup are commercially available transparent foils and glass: Melinex $401 \mathrm{CW}$ of $100 \mu \mathrm{m}$ thickness, Mylar A of $100 \mu \mathrm{m}$ thickness, Kapton HN300 of $76.2 \mu \mathrm{m}$ thickness, Lyreco polypropylene file pockets of $90 \mu \mathrm{m}$ thickness and soda lime glass slides of $1.1 \mathrm{~mm}$ thickness. The experimental details regarding inkjet printing of the $\mathrm{Ag}$ ink on Melinex and glass, as well as the entire OLED fabrication and characterization, are described elsewhere. ${ }^{33}$

\section{RESULTS}

We performed the haze measurement described above using red $(650 \mathrm{~nm})$, green $(532 \mathrm{~nm})$ and blue $(405 \mathrm{~nm})$ laser pointers on a variety of transparent films and noted the radius and angle of the pattern that was formed. Figure 5 provides an 
overview of the resulting image patterns. In each case the radius of the pattern was measured, the corresponding angle calculated and the difference $(\Delta)$ provided to the reference pattern. For the pristine PET and glass samples, almost no difference ( $\Delta$ up to $0.6^{\circ}$ ) to the blank image is seen, i.e. without any sample in front of the laser pointer. The respective PET and glass samples with the inkjet-printed Ag layer increase haze slightly ( $\Delta$ up to $0.8^{\circ}$ in the case of red and green light).

For comparison, two diffusing foils were included - Mylar, a polyester foil and a diffusing office foil used for documents, which both showed high haze $\left(\Delta\right.$ up to $\left.5.3^{\circ}\right)$. Finally, a polyimide foil shows low haze $\left(\Delta 0.8^{\circ}\right)$, although images are only visible for the red and green laser pointers, since the orange foil absorbs the blue light. It must be noted that the series of qualitative results are only comparable within one color series, and - as in the polyimide film - depend on the transmission of the sample to light of that particular color. Additionally, the patterns created by the blue laser pointer are systematically larger, since the blue light causes an emission of the optical brighteners used in many types of office paper.

Overall, the films quantified with low haze by the manufacturer - and that are visibly clear to the eye - confirm this property by only showing a slight deviation in pattern size from the reference. In many cases the deviation is below the $2.5^{\circ}$ haze threshold. For the noticeably less clear films (polyester and polypropylene) the findings are also in line with the optical perception of these two films. Finally, the inkjet-printed Ag films show only slightly larger haze than the pristine PET film and, as has been shown in our previous work, ${ }^{32}$ have lower haze than films containing ITO.

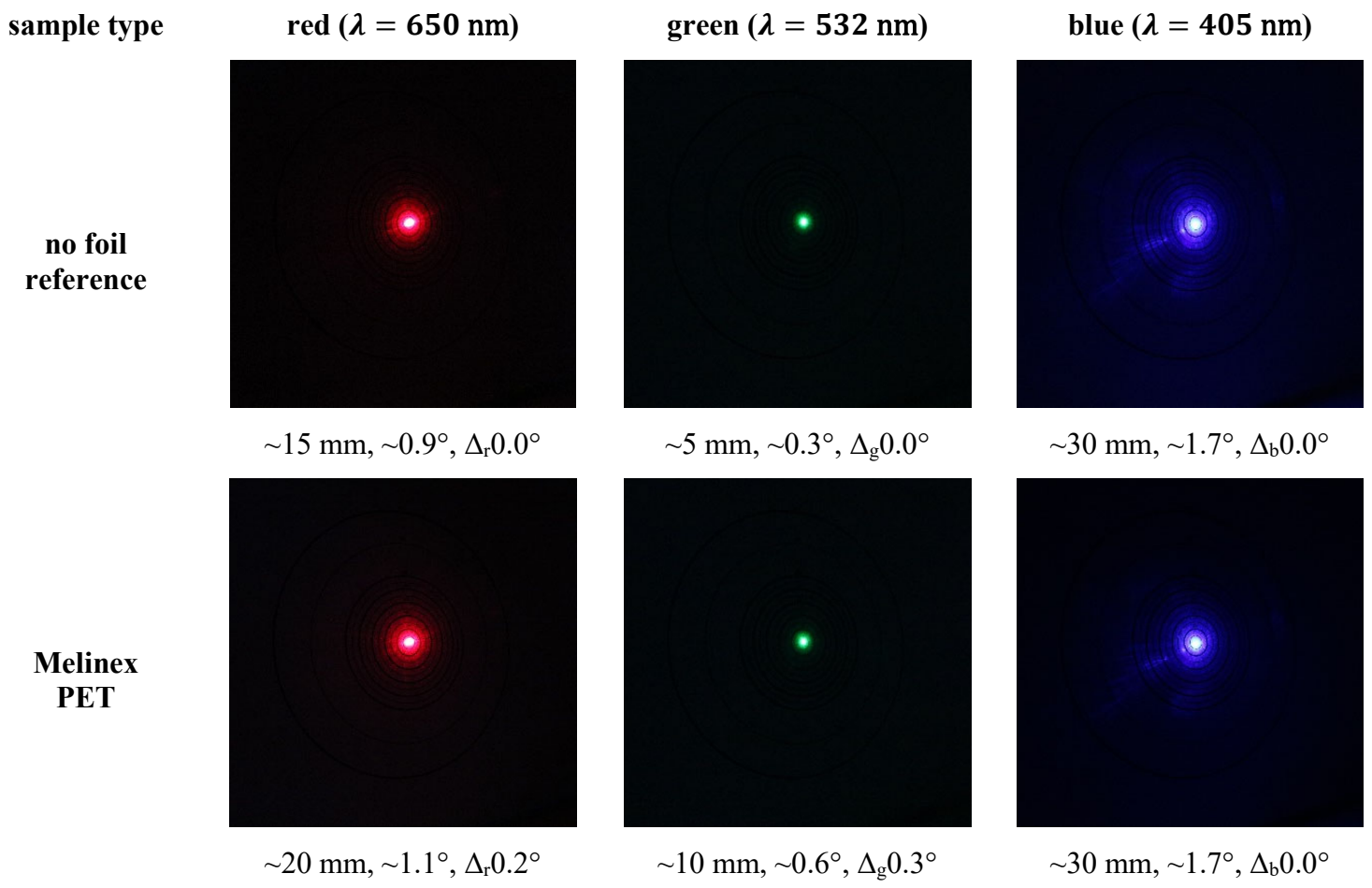


Ag ink

on PET

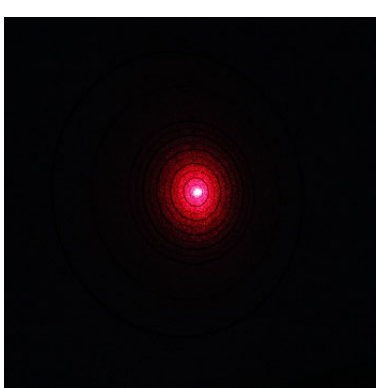

$\sim 30 \mathrm{~mm}, \sim 1.7^{\circ}, \Delta_{\mathrm{r}} 0.8^{\circ}$

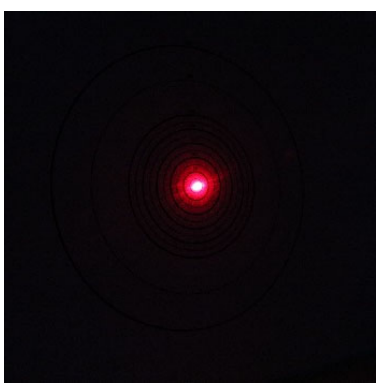

$\sim 20 \mathrm{~mm}, \sim 1.1^{\circ}, \Delta_{\mathrm{r}} 0.2^{\circ}$

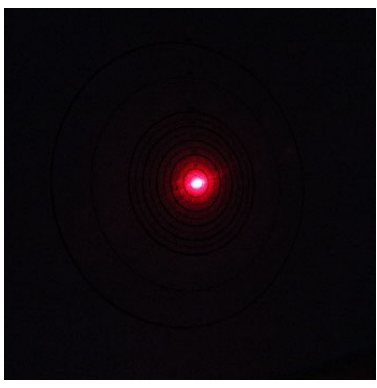

$\sim 20 \mathrm{~mm}, \sim 1.1^{\circ}, \Delta_{\mathrm{r}} 0.2^{\circ}$

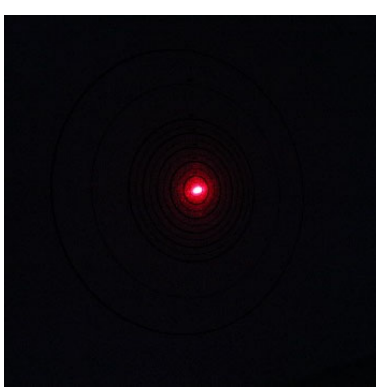

$\sim 15 \mathrm{~mm}, \sim 0.9^{\circ}, \Delta_{\mathrm{r}} 0.0^{\circ}$

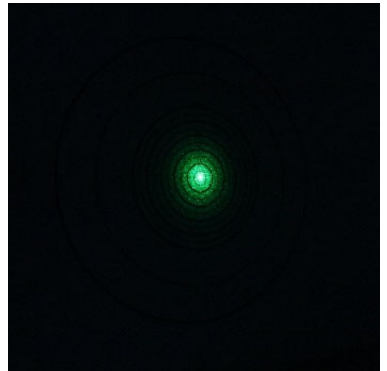

$\sim 20 \mathrm{~mm}, \sim 1.1^{\circ}, \Delta_{\mathrm{g}} 0.8^{\circ}$

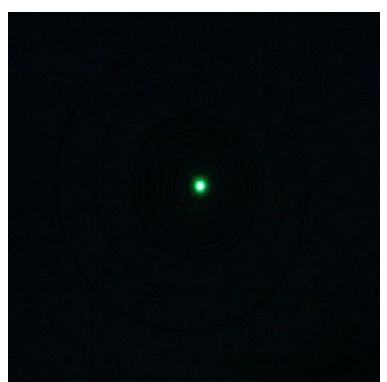

$\sim 5 \mathrm{~mm}, \sim 0.3^{\circ}, \Delta_{\mathrm{g}} 0.0^{\circ}$

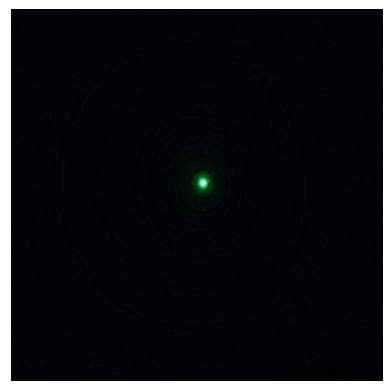

$\sim 5 \mathrm{~mm}, \sim 0.3^{\circ}, \Delta_{\mathrm{g}} 0.0^{\circ}$

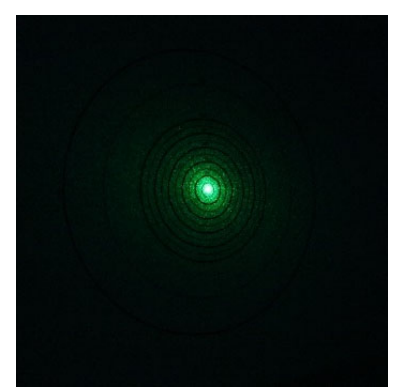

$\sim 50 \mathrm{~mm}, \sim 2.9^{\circ}, \Delta_{\mathrm{g}} 2.6^{\circ}$

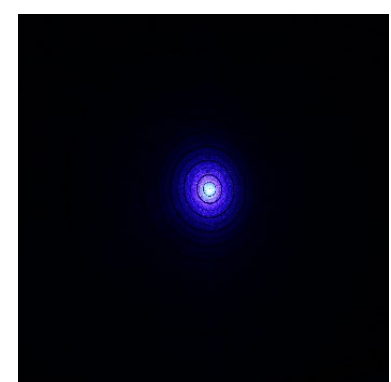

$\sim 35 \mathrm{~mm}, \sim 2.0^{\circ}, \Delta_{\mathrm{b}} 0.3^{\circ}$

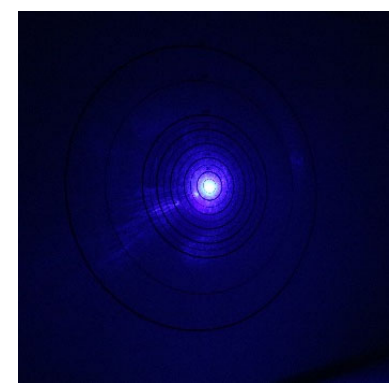

$\sim 40 \mathrm{~mm}, \sim 2.3^{\circ}, \Delta_{\mathrm{b}} 0.6^{\circ}$

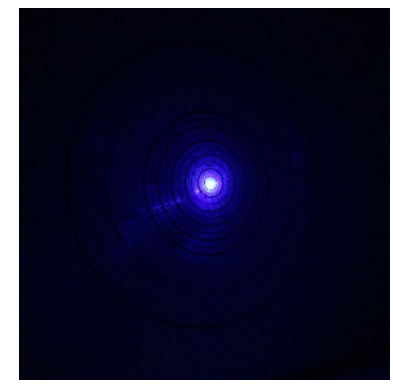

$\sim 40 \mathrm{~mm}, \sim 2.3^{\circ}, \Delta_{\mathrm{b}} 0.6^{\circ}$

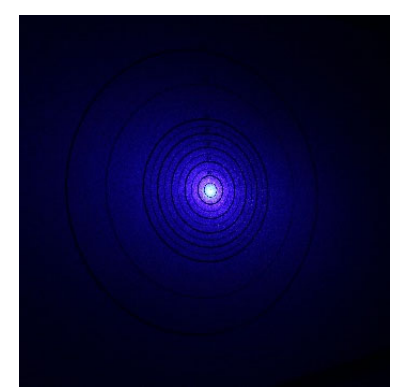

$\sim 50 \mathrm{~mm}, \sim 2.9^{\circ}, \Delta_{\mathrm{b}} 1.2^{\circ}$ 


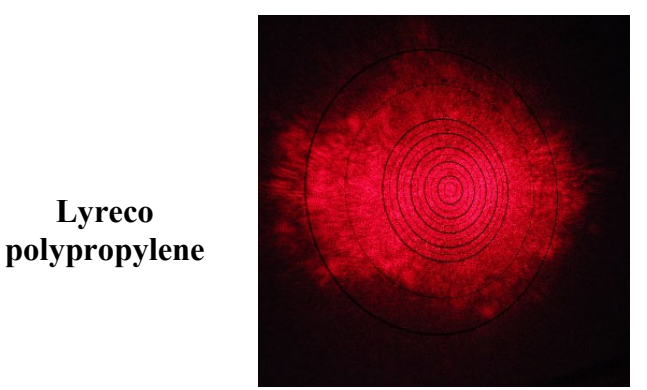

$\sim 100 \mathrm{~mm}, \sim 5.7^{\circ}, \Delta_{\mathrm{r}} 4.8^{\circ}$

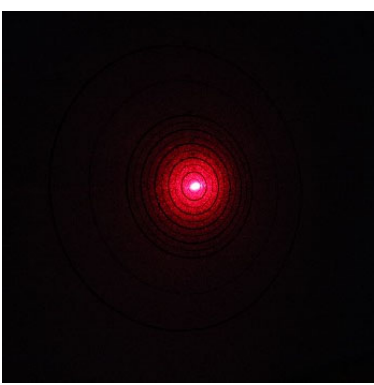

$\sim 30 \mathrm{~mm}, \sim 1.7^{\circ}, \Delta_{\mathrm{r}} 0.8^{\circ}$

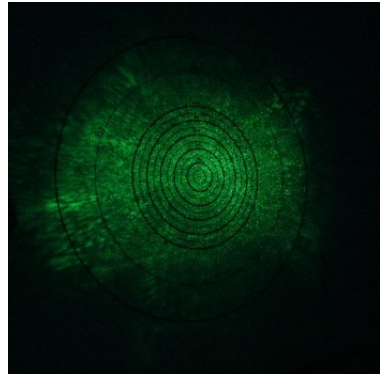

$\sim 100 \mathrm{~mm}, \sim 5.7^{\circ}, \Delta_{\mathrm{g}} 5.3^{\circ}$

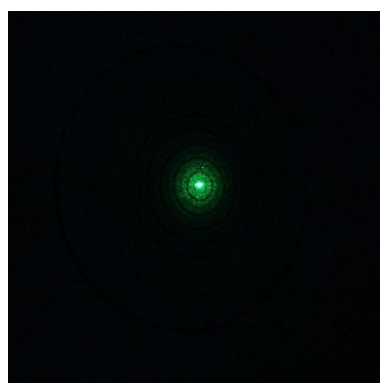

$\sim 20 \mathrm{~mm}, \sim 1.1^{\circ}, \Delta_{\mathrm{g}} 0.8^{\circ}$

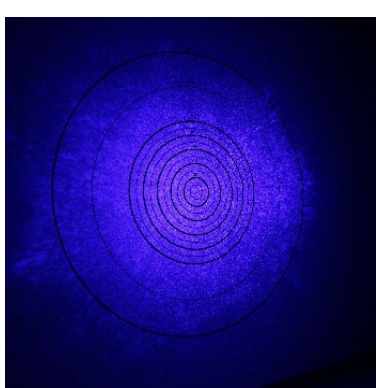

$>100 \mathrm{~mm},>5.7^{\circ}, \Delta_{\mathrm{b}} 5.0^{\circ}$

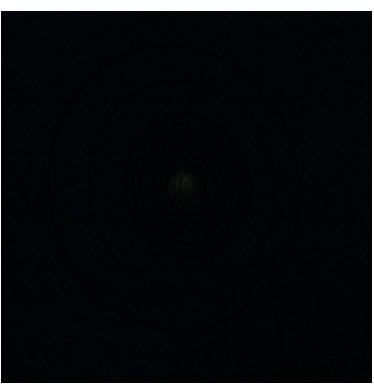

$\mathrm{n} / \mathrm{a}$

Figure 5. The overview of patterns achieved by shining a laser pointer with varying color through a series of transparent samples shows the low haze of the samples containing the printed Ag ink, since it is only marginally larger than the pristine PET film. The diffusing polypropylene film shows much larger patterns, indicative of a hazy material. The $\Delta_{\mathrm{r} / \mathrm{g} / \mathrm{b}}$ values refer to the difference in angle to the reference measurement of the same color.

\section{CONCLUSIONS}

Driven by the desire to understand how inkjet-printed Ag layers with distinct crystalline features influence haze, we have developed a simple setup to investigate this property. The setup provides qualitative results in order to determine the relative change of haze within a series of samples or in comparison to a blank reference. The technique can be complemented by quantitative measurements using a spectrophotometer or hazemeter, if the absolute haze values are required. The setup can be configured using components that are readily accessible in most research labs. Using this setup, we investigated the haze of a series of transparent substrates as well as of inkjet-printed transparent electrodes based on a Ag particle-free ink on rigid glass and flexible PET substrates. The transparent electrodes were implemented in flexible OLEDs to produce devices with high brightness and low haze.

\section{ACKNOWLEDGEMENTS}

This work was conducted in the framework of the Joint Lab GEN_FAB and was supported by the HySPRINT innovation lab at Helmholtz-Zentrum Berlin. This project has received funding from the European Union's Horizon 2020 research and innovation programme under grant agreement No 862410 (HI-ACCURACY).

\section{REFERENCES}

[1] Kim H, Gilmore C M, Piqué A, Horwitz J S, Mattoussi H, Murata H, Kafafi Z H and Chrisey D B 1999 Electrical, optical, and structural properties of indium-tin-oxide thin films for organic light-emitting devices Journal of Applied Physics 86 6451-61. 
[2] Chopra K L, Major S and Pandya D K 1983 Transparent conductors-A status review Thin Solid Films 102 $1-46$

[3] Luo M, Liu Y, Huang W, Qiao W, Zhou Y, Ye Y and Chen L sen 2017 Towards flexible transparent electrodes based on carbon and metallic materials Micromachines 8 1-16

[4] Peng C, Jia Z, Neilson H, Li T and Lou J 2013 In situ electro-mechanical experiments and mechanics modeling of fracture in indium tin oxide-based multilayer electrodes Advanced Engineering Materials 15 250-6

[5] F. Hermerschmidt, S. A. Choulis, and E. J. W. List-Kratochvil, "Implementing Inkjet-Printed Transparent Conductive Electrodes in Solution-Processed Organic Electronics," Advanced Materials Technologies, 4(5), (2019).

[6] Cho H, Yun C and Yoo S 2010 Multilayer transparent electrode for organic light-emitting diodes: tuning its optical characteristics Optics Express 183404

[7] L. Kinner, M. Bauch, R. A. Wibowo et al., "Polymer interlayers on flexible PET substrates enabling ultrahigh performance, ITO-free dielectric/metal/dielectric transparent electrode," Materials \& Design, 168, (2019).

[8] N. G. Semaltianos, "Thermally evaporated aluminium thin films,” Applied Surface Science, 183(3), 223 229 (2001).

[9] R. R. Søndergaard, M. Hösel, and F. C. Krebs, "Roll-to-Roll fabrication of large area functional organic materials,” Journal of Polymer Science Part B: Polymer Physics, 51(1), 16-34 (2013).

[10] L. Kinner, E. J. W. List-Kratochvil, and T. Dimopoulos, "Gentle plasma process for embedded silvernanowire flexible transparent electrodes on temperature-sensitive polymer substrates," Nanotechnology, 31(36), 365303 (2020).

[11]E. Georgiou, A. Savva, M. Neophytou et al., "Evaporation-free inverted organic photovoltaics using a mixture of silver nanoparticle ink formulations for solution-processed top electrodes," Applied Physics Letters, 105(23), (2014).

[12] W. Wu, "Inorganic nanomaterials for printed electronics: a review," Nanoscale, 9(22), 7342-7372 (2017).

[13] W. Yang, E. J. W. List-Kratochvil, and C. Wang, "Metal particle-free inks for printed flexible electronics," Journal of Materials Chemistry C, 7(48), 15098-15117 (2019).

[14] M. Vaseem, S.-K. Lee, J.-G. Kim et al., "Silver-ethanolamine-formate complex based transparent and stable ink: Electrical assessment with microwave plasma vs thermal sintering," Chemical Engineering Journal, 306, 796-805 (2016).

[15] Y. Choi, K. d. Seong, and Y. Piao, "Metal-Organic Decomposition Ink for Printed Electronics," Advanced Materials Interfaces, 6(20), (2019).

[16] A. Kamyshny, and S. Magdassi, "Conductive nanomaterials for printed electronics," Small, 10(17), 351535 (2014).

[17] Y. Galagan, E. W. C. Coenen, S. Sabik et al., "Evaluation of ink-jet printed current collecting grids and busbars for ITO-free organic solar cells," Solar Energy Materials and Solar Cells, 104, 32-38 (2012).

[18] Y. Galagan, B. Zimmermann, E. W. C. Coenen et al., "Current Collecting Grids for ITO-Free Solar Cells," Advanced Energy Materials, 2(1), 103-110 (2012).

[19]L. Kinner, S. Nau, K. Popovic et al., "Inkjet-printed embedded Ag-PEDOT:PSS electrodes with improved light out coupling effects for highly efficient ITO-free blue polymer light emitting diodes," Applied Physics Letters, 110(10), (2017).

[20] T. M. Eggenhuisen, Y. Galagan, A. F. K. V. Biezemans et al., "High efficiency, fully inkjet printed organic solar cells with freedom of design," Journal of Materials Chemistry A, 3(14), 7255-7262 (2015).

[21] W. Yang, F. Mathies, E. L. Unger et al., "One-pot synthesis of a stable and cost-effective silver particlefree ink for inkjet-printed flexible electronics," Journal of Materials Chemistry C, 8(46), 16443-16451 (2020).

[22]D. Li, W. Y. Lai, Y. Z. Zhang et al., "Printable Transparent Conductive Films for Flexible Electronics," Adv Mater, 30(10), (2018).

[23]F. Hermerschmidt, P. Papagiorgis, A. Savva et al., "Inkjet printing processing conditions for bulkheterojunction solar cells using two high-performing conjugated polymer donors," Solar Energy Materials and Solar Cells, 130, 474-480 (2014).

[24] S. M. Pozov, G. Schider, S. Voigt et al., "Up-scalable ITO-free organic light emitting diodes based on embedded inkjet-printed copper grids," Flexible and Printed Electronics, 4(2), (2019). 
[25]X. Lu, Y. Zhang, and Z. Zheng, "Metal-Based Flexible Transparent Electrodes: Challenges and Recent Advances," Advanced Electronic Materials, 7(5), (2021).

[26] O. Glushko, A. Klug, E. J. W. List-Kratochvil et al., "Monotonic and cyclic mechanical reliability of metallization lines on polymer substrates," Journal of Materials Research, 32(9), 1760-1769 (2017).

[27] O. Glushko, A. Klug, E. J. W. List-Kratochvil et al., "Relationship between mechanical damage and electrical degradation in polymer-supported metal films subjected to cyclic loading," Materials Science and Engineering: A, 662, 157-161 (2016).

[28] M. J. Cordill, O. Glushko, and B. Putz, "Electro-Mechanical Testing of Conductive Materials Used in Flexible Electronics," Frontiers in Materials, 3, (2016).

[29] L. Kinner, F. Hermerschmidt, T. Dimopoulos et al., "Implementation of Flexible Embedded Nanowire Electrodes in Organic Light-Emitting Diodes," physica status solidi (RRL) - Rapid Research Letters, 14(11), (2020).

[30] L. Kinner, T. Dimopoulos, G. Ligorio et al., "High performance organic light-emitting diodes employing ITO-free and flexible TiOx/Ag/Al:ZnO electrodes," RSC Advances, 11(28), 17324-17331 (2021).

[31]I. Genish, A. Irzh, A. Gedanken et al., "Coating dielectric substrates by plasma-reduction of metallic ions in solvents," Surface and Coatings Technology, 204(9-10), 1347-1352 (2010).

[32] M. Hengge, K. Livanov, N. Zamoshchik et al., "ITO-free OLEDs utilizing inkjet-printed and low temperature plasma-sintered Ag electrodes," Flexible and Printed Electronics, 6(1), (2021).

[33] ASTM International, [ASTM D1003-21, Standard Test Method for Haze and Luminous Transmittance of Transparent Plastics], West Conshohocken, PA (2021).

[34] International Organization for Standardization, [ISO 13468-1:2019 Plastics - Determination of the total luminous transmittance of transparent materials], Geneva (2019).

[35]F. Galeotti, W. Mróz, M. Catellani et al., "Tailorable perylene-loaded fluorescent nanostructures: a multifaceted approach enabling their application in white hybrid LEDs," Journal of Materials Chemistry C, 4(23), 5407-5415 (2016).

[36] W.-C. Liu, J. Hwang, A. Koo et al., “APMP Pilot Study on Transmittance Haze,” Journal of Physics: Conference Series, 972, 012023 (2018).

[37] B. Scharfe, S. Lehmann, T. Gerdes et al., "Optical and Mechanical Properties of Highly Transparent GlassFlake Composites,” Journal of Composites Science, 3(4), (2019).

[38]Z. Huang, C. Cai, X. Shi et al., "Leaf-mimicking polymers for hydrophobicity and high transmission haze," Proceedings of the Estonian Academy of Sciences, 66(4), (2016). 


\section{SUPPORTING INFORMATION}

Please print out this pattern at 1:1 scale (best on A3 paper) and affix to the wall $1000 \mathrm{~mm}$ from your sample.

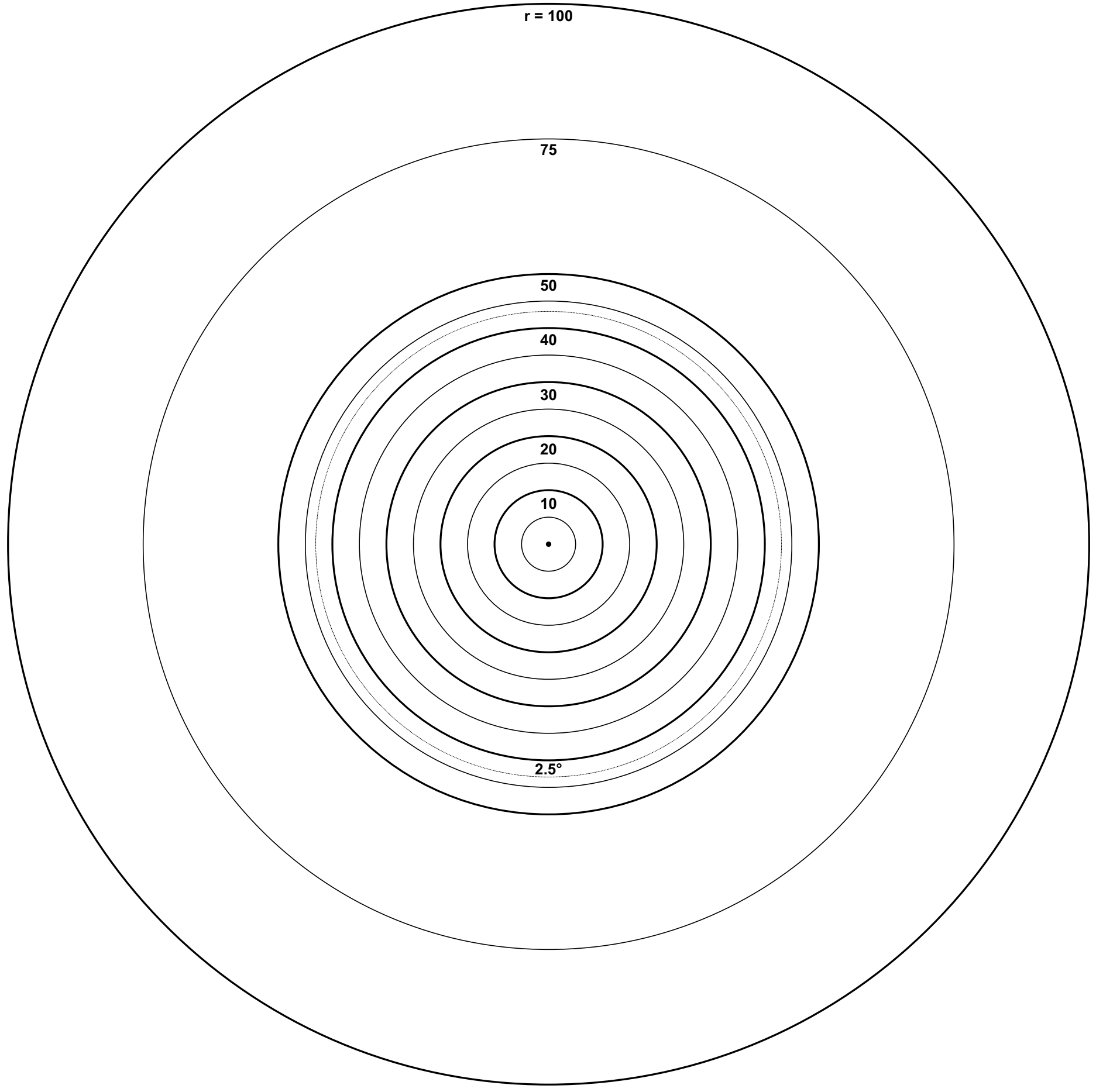

Proc. of SPIE Vol. 11808 118080B-11 